\title{
Sedna, 2004 VN112 and 2000 CR105: the tip of an iceberg
}

\author{
Rodney S. Gomes and Jean S. Soares \\ Observatório Nacional \\ Rua General José Cristino 77, CEP 20921-400, Rio de Janeiro, RJ, Brazil \\ email: rodney@on.br
}

\begin{abstract}
We review two main scenarios that may have implanted Sedna, 2004 VN112 and 2000 CR105 on their current peculiar orbits. These scenarios are based on perihelion lifting mechanisms that acted upon primordial scattered icy bodies. Supposing that the Sun was formed in a dense star cluster and that the gas giants were also forming while the cluster was still dense, an inner Oort cloud that includes Sedna at its inner edge could have been formed by the circularization of icy leftovers orbits scattered by the gas giants. A putative planetary mass solar companion can also produce a similar population of icy bodies through a perihelion lifting mechanism induced by secular resonances from the companion. A third scenario also dependent on a primordial dense cluster may contribute to adding a significant number of extrasolar icy bodies to the main solar component of the population created by the cluster model. These extrasolar objects are transferred to Sun orbits from the scattered disk of passing stars that were numerous in the dense primordial environment. We compare the scenarios as to the orbital distribution of the induced populations as well as their total mass. We conclude that both the cluster model and the solar companion model can produce icy body populations consistent with Sedna's orbit. It is also quite possible that this inner Oort cloud may be composed of roughly one tenth of extrasolar objects.
\end{abstract}

Keywords. Sedna, Oort cloud, star cluster, solar companion

\section{Introduction}

Gladman et al. (2008) presents a comprehensive nomenclature for trans-Neptunian orbits by which Sedna, 2004 VN112 and 2000 CR105 are classified as detached objects. These icy bodies are those nonscattering transneptunian objects with large eccentricities $(e>0.24)$ and not so far away that external influences are important to their current dynamics $(a<2000 A U)$. On the same nomenclature a scattering object is that which is currently scattering actively off Neptune. Although it is usually accepted that a detached object was once a scattering object that for some dynamical process had its perihelion lifted (see Gomes et al. 2005a, Gladman et al. 2002), it is also possible that some detached objects may have another origin. Nevertheless Gomes et al. (2005a) show that resonant perturbations from the outermost planets are very effective in lifting scattered objects perihelia, for those with semimajor axes not larger than roughly 200AU. With this in mind we might conjecture with some confidence that detached objects are formerly scattered objects whose perihelia were lifted by the coupling of mean motion and Kozai resonances with an outer planet, usually Neptune. If we thus define a detached object, we are forced to leave undefined three trans-Neptunian objects that cannot (or can hardly, in the case of 2000 CR105) experience an increase of their perihelion by the sole perturbations of the outer planets. A scenario for their origin must therefore be sought 
elsewhere beyond the perturbations of the known Solar System. The main scenarios invoke either conditions that prevailed in a primordial Solar System or conditions that may still be present in the Solar System. The latter case stands for the solar companion scenario (Gomes et al. 2006) and the former case refers to the scenario of a primordial dense star cluster in which the Sun would be embedded. In this case torques from this dense environment would increase the perihelia of icy bodies being scattered outwards by the giant planets (Brasser et al. 2006, 2007, 2008). This latter scenario is also consistent with the transfer of several extrasolar icy bodies from other stars to circumsolar orbits. For this we just have to assume that the other stars were experiencing a similar process of planetesimals scattering by their icy giants, which is a quite reasonable hypothesis. In this sense, this capture scenario will be considered as a third one and described in Section 2, in which we also review the first two scenarios. With respect to the cluster model, we have produced our own data following Brasser et al. (2006). In Section 3, we compare scenarios so as to assess their relative plausibilities. We estimate the mass of the populations produced by each scenario in Seccion 4 and conclusions are drawn in Section 5 .

\section{Scenarios for the formation of Sedna population}

Galactic tides induce strong long period variations to the eccentricities of objects in distant orbits around the Sun. This process can thus create a cloud of comets in the Oort cloud as well as send an icy body back to the inner Solar System as a long period comet. The closest distance where galactic tide can be effective in increasing an icy body perihelion to that of Sedna is roughly 4000 AU. A much stronger tide would be necessary to raise Sedna's perihelion, with a semimajor axis at 510 AU. This condition could be satisfied by the torque from close passing stars and the gas in a primordial star cluster dense environment where the Sun would be embedded (Brasser et al. 2006). The inner edge of the thus formed 'inner Oort cloud' will be as closer to the Sun as larger is the density of the cluster. Figure 1 shows the distribution of semimajor axes and perihelion distances of planetesimals scattered by Jupiter and Saturn with raised perihelia by the tidal effect of a star cluster where the Solar System would be embedded. In a primordial star cluster, experiencing gravitational effects from passing stars and the gas, many planetesimals will experience perihelion increases. To build this figure, we followed the procedures described in Brasser et al. 2006, which we recommend for details on the method. In principle, from an observational basis, one would expect that the right population created by the primordial cluster should have Sedna and 2004 VN112 (eventually 2000 CR105) at the population inner edge. From Fig. 1, we estimate that the best central density that could create such a population would be between $10^{4}$ and $10^{5}$ $M_{\text {Sun }} / p c^{3}$. One should note however that those $a \times q$ distributions also depend on the orbit of the Sun inside the cluster. A Sun that inhabited the densest parts of the cluster would produce in the end a Sedna population closer to the Sun than a Sun with an orbit in a less dense part of the cluster. The cluster model yields a fairly likely scenario since it is probable that the Sun like most stars also formed in such a dense environment. Another reasonable supposition implied by this scenario is that at this primordial time the Solar System formed its gas giants and planetesimals leftovers would experience deep encounters with the planets, thus increasing their semimajor axes and placing them at the right distance to have their perihelia increased by the tides from the cluster.

Another reasonable conjecture related to the scenario of the Sun in a primordial dense star cluster is that, like the Sun, the other cluster stars must experience a similar planetary formation and scattering of icy leftovers. A cloud of scattered (lifted perihelia) 

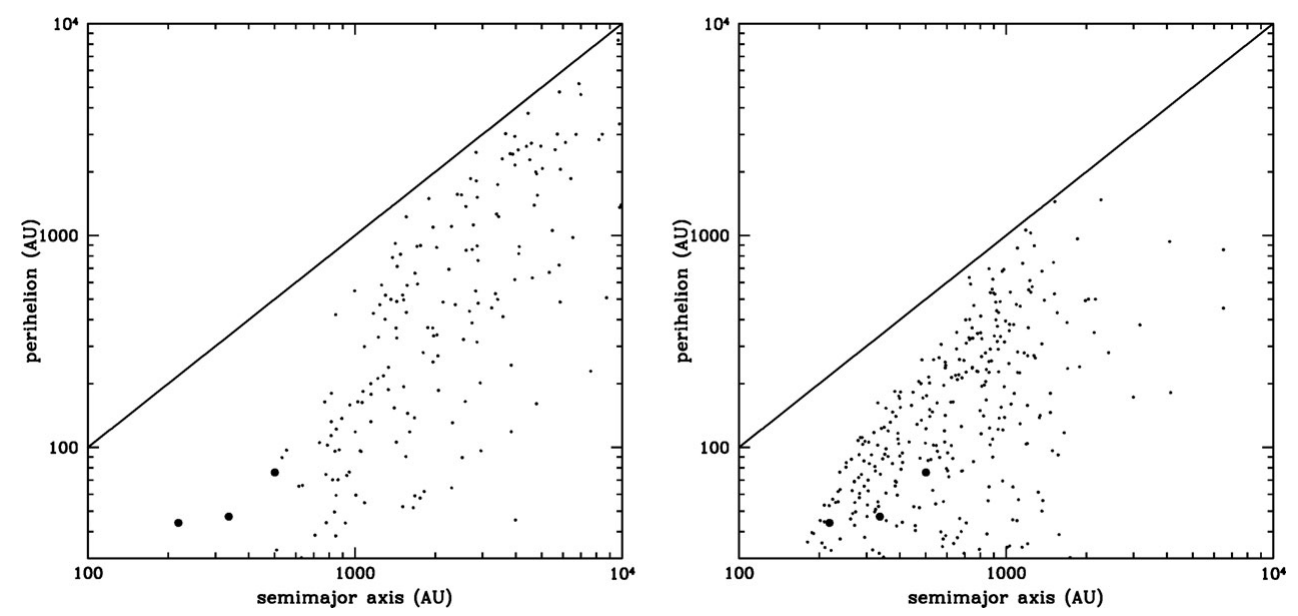

Figure 1. Distribution of semimajor axes and perihelion distances for a population of icy bodies created by the cluster model. Left panel: cluster central density $10^{4} M_{S u n} / p c^{3}$, right panel: cluster density $10^{5} M_{S u n} / p c^{3}$. Sedna, 2004 VN112 and 2000 CR105 are represented by large circles.

planetesimals around a close passing star must transfer some of its objects to a Sun orbit. Fig. 2 shows the distribution of semimajor axes and perihelia of planetesimals (gray circles) transferred from a passing star orbit to a Sun orbit. The star has one solar mass and comes as close as 1000 AU to the Sun with $v_{\infty}=0.6 \mathrm{~km} \mathrm{~s}^{-1}$. The dots in this figure comes from the same simulation as those in Fig. 1 (less dense case). We have however taken particles from more than one time in the original simulation to increase the number of transferred objects and get a nicer statistics for the $a \times q$ distribution for the extrasolar bodies. So Fig. 2 allows for the comparison of the relative amount of planetesimals mass transferred to the Oort cloud from the inner Solar System with the mass from an extrasolar origin. We usually get roughly $10 \%$ of extrasolar objects with respect to solar planetesimals, for a star like the one considered in this example. On the other hand, the extrasolar icy bodies population usually have an inner edge closer to the Sun than that from solar origin. It must be noted that the star considered here is consistent with a cluster star orbit that induced the distribution of icy bodies orbits from the inner Solar System, so that the dots and gray circles in Fig. 2 are comparable. This example is mostly a proof of concept since we must do a more complete simulation with a series of close passages of stars with their scattered disks. Each star may leave some extra material around the Sun but also eject some of the already captured planetesimals from other stars.

A third scenario is based on the perturbation of a putative planetary mass solar companion (Gomes et al. 2006). At remote distances from the Sun, the angular displacement of objects around the Sun is very slow. This induces secular resonances on other distant objects. Figure 3 shows the distribution of semimajor axes and perihelion distances of planetesimals scattered by the major planets and gravitationally perturbed by a solar companion with $10^{-4}$ Earth mass, and semimajor axis, eccentricity and inclination respectively $1500 A U, 0.4$ and $40^{\circ}$. This example is taken from a simulation where particles have mass and induce a migration on the giant planets. Different solar companions yield similar effects provided $\rho=M / b^{3}$ is about the same, where $b$ is the companion semiminor axis and $M$ its mass. In other words, for a distant massive solar companion, there is a not so distant and less massive companion that yield similar induced $a \times q$ distributions. The 


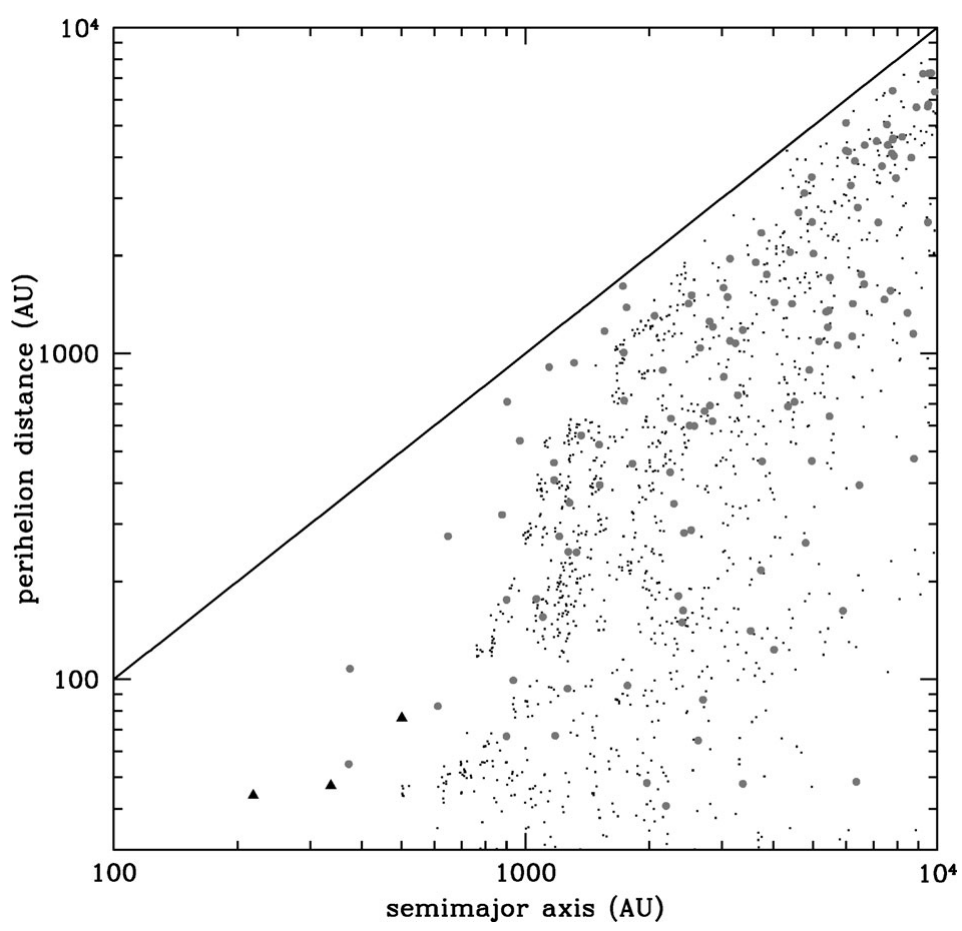

Figure 2. Distribution of semimajor axes and perihelion distances for a population of icy bodies created by the cluster model (small dots) added with a population of extrasolar objects transferred to a Sun orbit from the scattered disk of a passing star (gray circles). Sedna, 2004 VN112 and 2000 CR105 are represented by triangles.

solar companion model, differently from the cluster model, requires that the companion perturbations be effective for a much longer time than a star cluster's lifetime. This is not a problem, since it is supposed that the solar companion is there for the solar system age. On the other hand, existing for the solar system age is not necessarily required for the solar companion. Some hundreds of million years are sufficient to increase the perihelia of scattered objects. Thus a putative scattered planet (of roughly an Earth mass) could increase scattered objects perihelia to produce a Sedna population. This scattered planet could be eventually ejected from the Solar System. This hypothesis was also invoked by Lykawka and Mukai (2008) to explain other features of transneptunian objects orbits. However the probability of a specific scattered object staying for some hundred million years as a scattered/detached object is quite low (roughly $<1 \%$ ). Another possible origin of a solar companion comes from the star cluster model. In fact one of the objects scattered by the gas giants in the primordial cluster embedded solar system could be a planet. If there was originally one such planet the probability that it is still orbiting the Sun is given by the relative amount of perihelion increased bodies, which is near $10 \%$. Also the extrasolar objects eventually captured by the Sun as described by scenario 2 above might also include an extrasolar planetary size body, with the same probability as the total amount of extrasolar bodies transferred to the Sun from a star to the amount of scattered objects by the same star. And finally, a floating planet could be captured by the Sun in the dense cluster environment in a three-body encounter (Sun + star + planet). This floating planet might have just been ejected by a star system, what is a likely event in a primordial planetary system. 


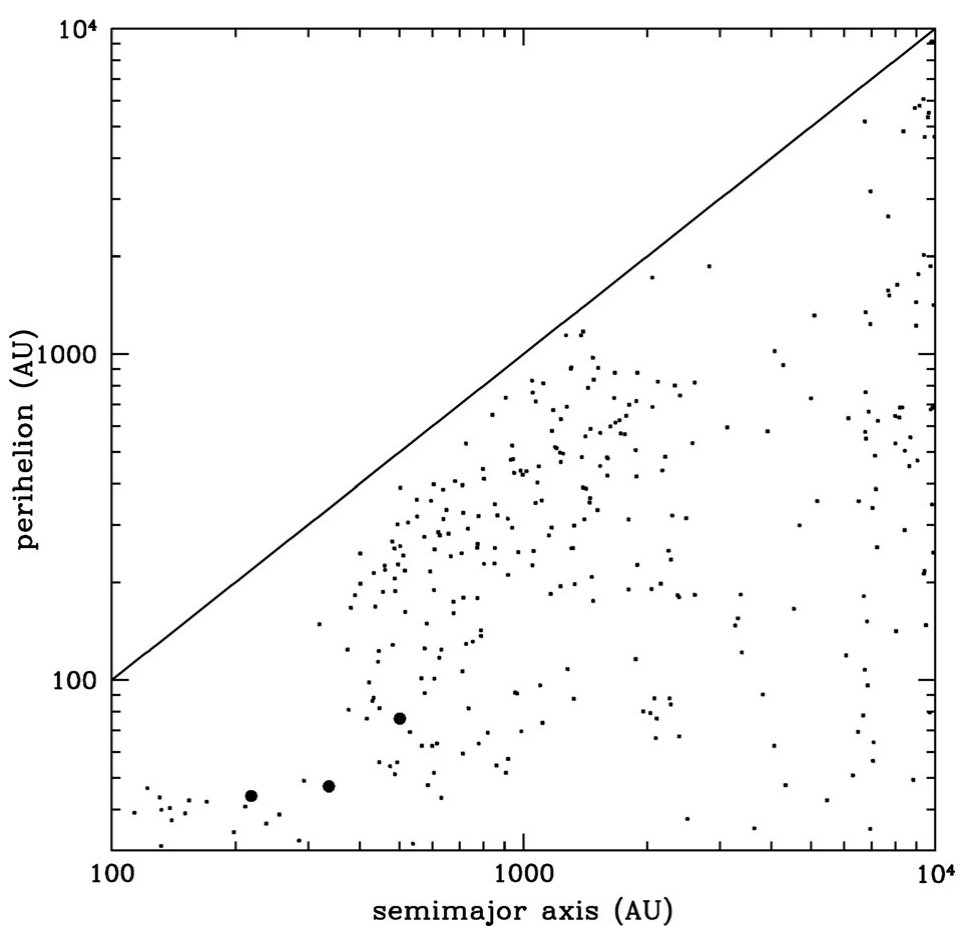

Figure 3. Distribution of semimajor axes and perihelion distances for a population of icy bodies created by the solar companion model. The companion mass is $10^{-4}$ solar mass and its semimajor axis, eccentricity and inclination are $1500 A U, 0.4$ and $40^{\circ}$. Sedna, 2004 VN112 and 2000 CR105 are represented by large circles.

It must be also noted that differently from the cluster scenario, the objects scattered by the solar system planets and deposited in the inner Oort cloud belong to a not so primordial time. These icy bodies would have been preferentially scattered by the icy giants during planetary migration in a planetesimal disk, possibly after the Late Heavy Bombardment (Gomes et al. 2005b). This is an important difference since in this case, there not being a gas disk around the Sun, small (visible comet size) bodies could also fill the inner Oort cloud, what would be prevented in a more primordial scenario where gas drag would not allow smaller icy bodies $(<2 \mathrm{~km}$ radius $)$ to reach distances as far as Sedna's distance from the Sun (Brasser et al. 2007). This is an interesting feature of the companion model, since Kaib \& Quinn (2009) claims that the inner Oort cloud could be an important reservoir of LPC's. The mechanism by which these comets follow a path that will make them eventually visible could in principle also be followed by an object from the much inner Oort cloud represented by the putative Sedna population. In the solar companion model Sedna's population is not fossilized but their objects are still moving in perihelion, what turns them eventually Neptune crossers again.

\section{Comparison between scenarios}

Comparing Figs. 1 and 3, it is noticeable that both distributions are similar. Although the inner edges of the populations in Fig. 1 and Fig. 3 are different, a suitable choice of cluster densities can place the inner edge from the cluster model at a more consistent position with the observation of Sedna. So in principle both models are undifferentiated if 

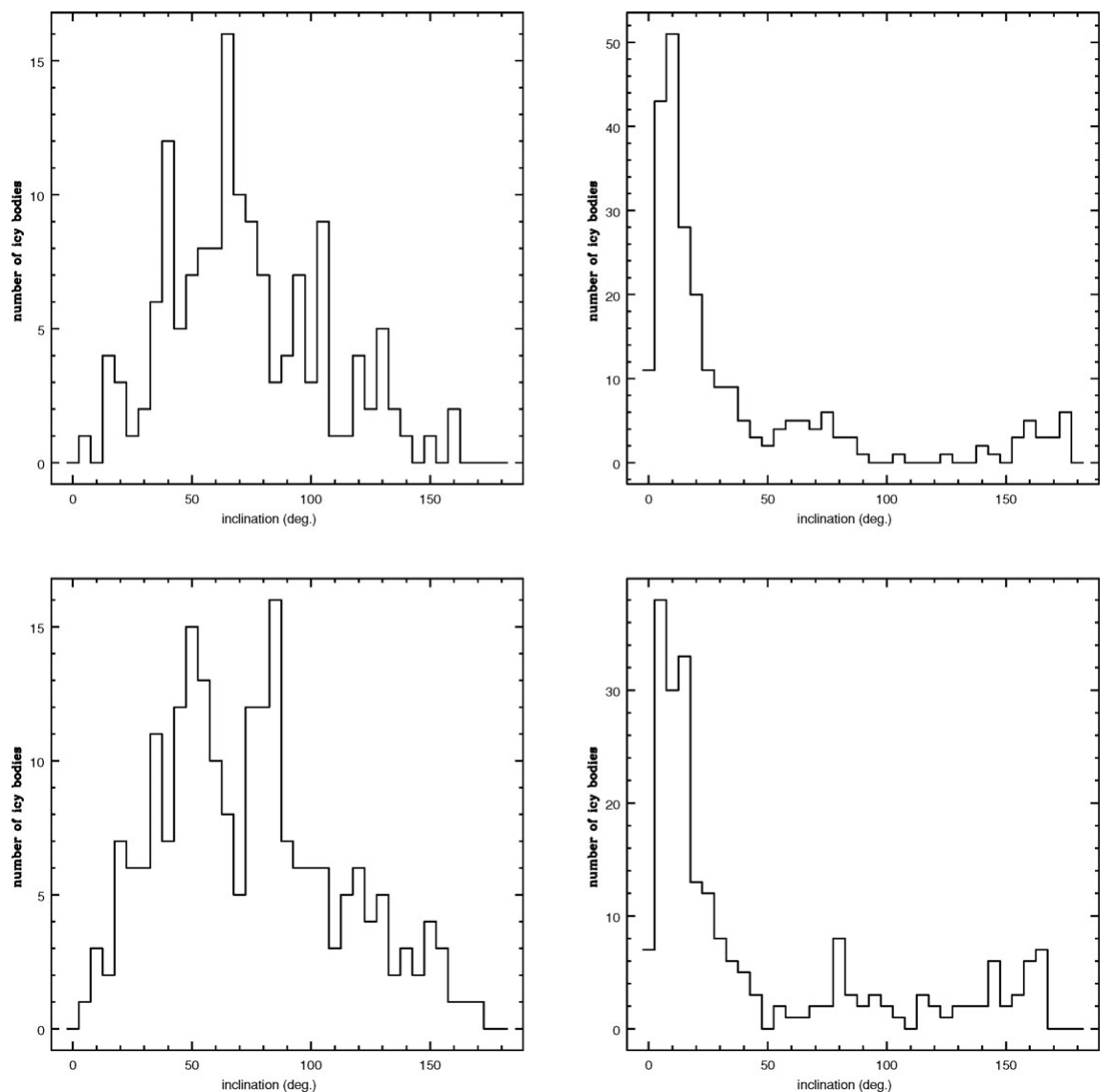

Figure 4. Histograms showing the distribution of inclinations of the icy bodies: upper left: from the cluster model with central density $10^{4} M_{S u n} / p c^{3}$, upper right: companion model with companion's inclination $i_{c}=0^{\circ}$, lower left: companion model for $i_{c}=45^{\circ}$, lower right: companion model for $i_{c}=90^{\circ}$.

we just compare their distributions in semimajor axis and perihelion distances. Another important orbital parameter for the icy bodies orbital distribution is the inclination. Fig. 4 shows a histogram of inclinations for the cluster model and for the companion model, in which case we consider three different inclinations for the companion, $0^{\circ}, 45^{\circ}$ and $90^{\circ}$. We see that both for the cluster model and the companion model with the companion inclination at $45^{\circ}$ the distributions of inclinations do not essentially differ peaking for inclinations just below $90^{\circ}$. However if the companion has an orbital inclination near $0^{\circ}$ or $90^{\circ}$ the distribution of inclination for Sedna's population peaks for $i<20^{\circ}$. This is an interesting feature to be taken into account when new Sedna-like objects are discovered. It is noteworthy that Sedna's inclination is $12^{\circ}$ and was discovered in a all-sky survey (Brown et al. 2004), what would suggest a low inclination Sedna population. 2004 VN112 and 2000 CR105 have inclinations respectively at $25^{\circ}$ and $23^{\circ}$, which are also fairly low. We must however await for new discoveries for a better judgment of this point.

We have developed a basic observational simulator in order to search for the most consistent model and the best parameters for that model. The simulator works as follows:

- We choose a population created by one of the above scenarios.

- We randomly choose an orbit within the population. 

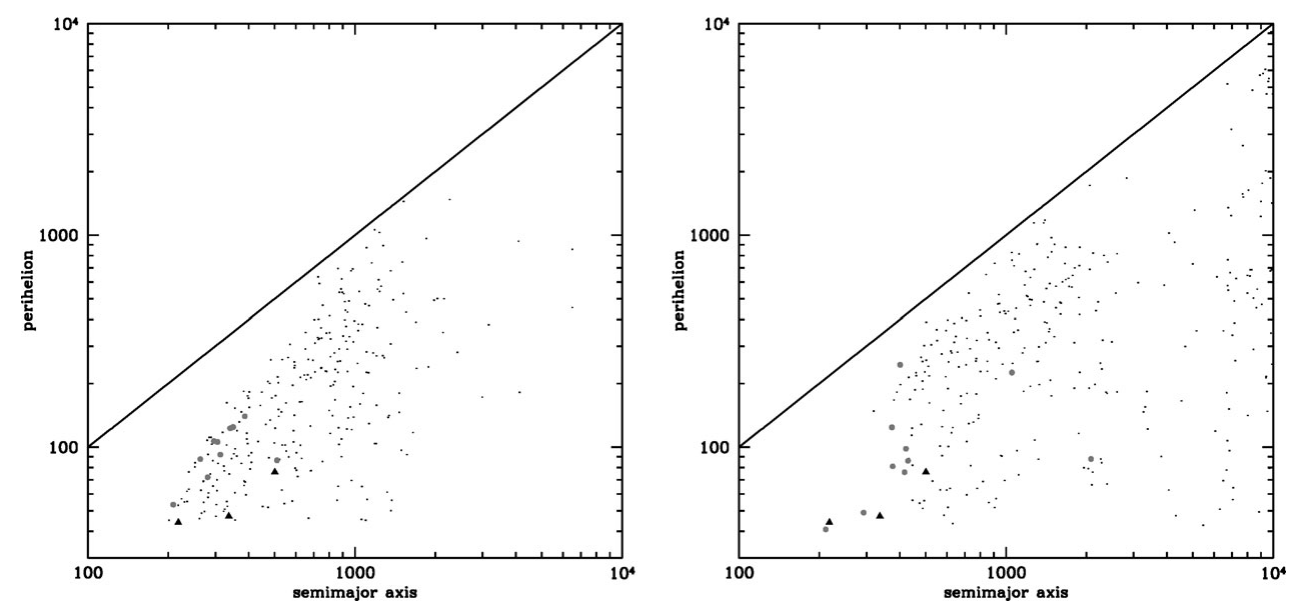

Figure 5. First ten 'observed objects' by the application of the observational simulator (gray circles). Left: for the cluster model with central density $10^{5} M_{S u n} / p c^{3}$, right: for the companion model with parameters as in Fig. 3. Sedna, 2004 VN112 and 2000 CR105 are represented by large triangles.

- We randomly choose a mean longitude for that orbit.

- We randomly choose a size for the body from a size distribution.

- Given an albedo, and the already computed size and distances of the body from the Sun and the Earth, we have its visual magnitude.

- If this magnitude is smaller that a given upper limit, the object is considered as observed.

- We can also limit the range of latitudes from which the observation was made.

Fig. 5 shows the first 10 observed objects from the cluster and companion models. For compatibility, we considered all objects with $a>200 A U$ and $q>45 A U$. We note that the simulator observations better accounts for the companion model. This should not be taken too rigorously since for the moment we have more examples with the companion model than for the cluster model, and a more fine-tuned cluster density may yield a better $a \times q$ distribution for the cluster model.

Another interesting application of the simulator can be made for the extrasolar objects model. In this case we put together the population created by the cluster model with an extrasolar population transferred from a star that passed near the Sun with orbital parameters compatible with the cluster parameters that generated the solar objects, so that both populations can be consistently placed together for an observational simulation. Applying the simulator several times we notice that although the extrasolar component of the population has several members closer to the Sun than the closest member from the solar component, an extrasolar object is first observed in about one time out of ten, showing that Sedna has most probably a solar origin.

\section{The size of Sedna population}

When Sedna was discovered (Brown et al. 2004) a mass of about 5 Earth masses was estimated for the population to which Sedna belongs. A more recent estimate predicts a total mass of about ten times the Kuiper belt mass (Schwamb et al. 2009). This yields roughly 0.1 to 1 Earth mass for Sedna population but possibly closer to the lower limit. Since these estimates are deduced from observational arguments, this inferred mass 
refers in fact to objects in orbits similar to Sedna's (with respect to semimajor axis and perihelion distance), so that would only represent the inner portion of the population coming from one of the theoretical models above presented. On the other hand, those models produce about 1-2 Earth masses in Sedna's population. We can thus conclude that the most recent observational based estimate of Sedna's population is more consistent with the populations created by any of the available models. In fact if we consider just the objects with $400 A U<a<800 A U$ and $50 A U<q<200 A U$, in the population shown in Fig. 3, that will stand for $3 \%$ of the total population, thus about 0.03 Earth masses, roughly one order of magnitude below the observational mass estimate. In any case, observational considerations associated with formation models suggest that with respect to Sedna, 2004 VN112 and 2000 CR105, we presently see just the tip of an iceberg. Since models consistently create a population distributed in a wide range of semimajor axes and perihelion distances and we now observe just three objects presumably at the inner edge of the population, it is thus likely that we are now just seeing a very small portion of the total population.

\section{Conclusions}

The orbits of Sedna, 2004 VN112 and to a lesser degree 2000 CR105 demand an explanation that goes beyond the perturbations from the known Solar System. These orbits might be considered close to the inner edge of an inward extended Oort cloud. Differently from the classical Oort cloud whose inner edge is formed at about $4000 \mathrm{AU}$ due to galactic tides, Sedna's population would start at roughly 300 - 500 AU. Galactic tides cannot account for such a close perihelion lifting, but an early dense environment where the Sun was supposedly formed can produce a tidal effect of much larger magnitude that could explain Sedna's orbit. The high plausibility of the Sun forming in such a high density environment associated with an induced orbital distribution of perihelion-lifted icy bodies consistent with Sedna's orbit gives the dense star cluster model a high degree of plausibility. A competing scenario requires that a planetary-mass solar companion would orbit the Sun in a distant orbit. Although also yielding a consistent orbital distribution for the icy bodies, it is not a strong supposition that a companion should exist or have existed orbiting the Sun. It must be noted on the other hand, that present observational methods or other indirect detection methods cannot presently discard a solar companion with the mass/distance compatible with the production of Sedna's population. Also the dense primordial star cluster scenario could be responsible for the placement of a planet at a distant orbit around the Sun, both by a solar origin or through an extrasolar origin. Although we do not need to invoke a solar companion to explain Sedna's orbit, its putative existence, although not very likely, is however far from negligibly probable, say some $10 \%$ chance of existing just for the known processes that can place a companion in a Sun orbit. So the companion scenario must be considered seriously as new Sedna-like bodies are discovered. The dense cluster scenario can also produce a subpopulation of extrasolar icy bodies. We just have to suppose that the passing stars, like the Sun, also carried a scattered/detached population of icy bodies. A simulation of a Sun mass star coming as close as $1000 \mathrm{AU}$ to the Sun and with $v_{\infty}=0.6 \mathrm{~km} \mathrm{~s}^{-1}$ showed that roughly $10 \%$ of its scattering/detached population (simulated like the Sun scattered population at that time) is transferred into orbits around the Sun. This subpopulation has its closest members to the Sun consistently inside the inner edge of the solar population. More complete simulations are needed to better determine the real fraction of extrasolar bodies with respect to solar ones and also their relative orbital distributions. Although this extrasolar component is not particularly large, it is however far from negligible and has 
a likeliness comparable to that of the solar component for the same scenario, so that one would expect to find one extrasolar icy body in Sedna's putative population after 10 solar icy bodies discoveries. Undoubtedly, new discoveries of transneptunian objects with large semimajor axes and perihelion distances will be extremely helpful is disclosing the invisible part of the 'iceberg' so that we can better understand the origin of Sedna and its correlates.

\section{References}

Brasser, R., Duncan, M. J., \& Levison, H. F. 2006, Icarus, 184, 59

Brasser, R., Duncan, M. J., \& Levison, H. F. 2007, Icarus, 191, 413

Brasser, R., Duncan, M. J., \& Levison, H. F. 2008, Icarus, 196, 274

Brown, M. E., Trujillo, C., \& Rabinowitz, D. 2004, ApJ, 617, 645

Gladman, B., Holman, M., Grav, T., Kavelaars, J., Nicholson, P., Aksnes, K., \& Petit, J-M. 2002, Icarus, 157, 269

Gladman, B., Marsden, B. G., \& VanLaerhoven, C. 2008, in: M. A. Barucci, H. Boehnhardt, D. P. Cruikshank \& A. Morbidelli (eds.), The Solar System Beyond Neptune (Tucson: The University of Arizona Press), p. 43

Gomes, R. S., Gallardo, T., Fernàndez, J. A., \& Brunini, A. 2005, CeMDA, 91, 109

Gomes, R., Levison, H. F., Tsiganis, K., \& Morbidelli, A. 2005, Nature, 7041, 466

Gomes, R. S., Matese, J., \& Lissauer, J. 2006, Icarus, 184, 589

Kaib, A. K. \& Quinn, T. 2009, Science, 325, 1234

Likawka, P. K. \& Mukai, T. 2008, AJ, 135, 1161

Schwamb, M. E., Brown, M. E., \& Rabinowitz, D. 2009, ApJ, 694, L45 